BIO Web of Conferences 2, 01001 (2014)

DOI: $10.1051 /$ bioconf/20140201001

(C) Owned by the authors, published by EDP Sciences, 2014

\title{
Planetary Environments: Scientific Issues and Perspectives
}

\author{
Th. Encrenaz ${ }^{1}$ \\ ${ }^{1}$ LESIA, Observatoire de Paris, CNRS, 5 place Janssen, 92195 Meudon, France
}

\begin{abstract}
What are the planetary environments where conditions are best suited for habitability? A first constraint is provided by the presence of liquid water. This condition allows us to define two kinds of media: (1) the atmospheres of solid (exo)planets with a temperature typically ranging between $0^{\circ} \mathrm{C}$ and $100^{\circ} \mathrm{C}$, and (2) the interiors of icy bodies (outer satellites or possibly exosatellites) where the pressure and temperature would fit the liquid phase region of the water phase diagram. In the case of Mars, significant progress has been achieved about our understanding of the history of liquid water in the past, thanks to the findings of recent space missions. The study of the outer satellites is also benefiting from the on-going operation of the Cassini mission. In the case of exoplanets, new discoveries are continuously reported, especially with the Kepler mission, in operation since 2009. With the emergence of transit spectroscopy, a new phase of exoplanets' exploration has started, their characterization, opening the new field of exoplanetology. In the future, new perspectives appear regarding the exploration of Mars, the giant planets and exoplanets, with the ultimate goal of characterizing the atmospheres of temperate exoplanets.
\end{abstract}

\section{How to search for traces of life in planetary environments?}

The quest for life in the Universe is as old as humanity. The question of the plurality of worlds was debated already by the Greek philosophers, and became again very active in the Copernicus era. Astronomers were actively involved in the controversy, in particular at the end of the XIX ${ }^{\text {th }}$ century, when Schiaparelli's discovery of "canali" at the surface of Mars was erroneously interpreted as the signature of intelligent life; the myth, although questioned by several astronomers including Antoniadi, lasted until the 1960s, when the first images returned by the space probes demonstrated that the canali were no more than an optical illusion. The possible existence of other planetary systems outside the solar system has been also a matter of discussion since Antiquity. For astronomers, the situation has radically changed in 1995, with the first discovery of a planet around a solar-type star. Today, early 2013, we know more than 850 exoplanets around nearby stars, some of them in multiple systems, and over two thousand candidates detected by the Kepler satellite. We now know that the solar system is not unique, but we have also discovered that exoplanets exhibit a huge variety of physical and orbital properties, often very different from the solar-system planets. In this context, the quest for extraterrestrial life, within the solar system and beyond, is more pertinent and vivid as ever.

\subsection{The search for liquid water}

How can we search for life, or traces of life, in solar system objects or elsewhere? The question is difficult, since we have no evidence yet about how life appeared on Earth. Did it emerge in the deep 
oceans, in the vicinity of hydrothermal vents, or was it brought from outside, through meteoritic or micrometeoritic impacts? Although we cannot answer this question, on the basis of what we know about terrestrial life, we can try to define an adequate set of favorable conditions. We do not know if these conditions are necessary in any case for life to emerge, but we know that they were essential for the appearance and development of life on Earth. By analogy, we will concentrate on these three conditions: (1) the presence of liquid water; (2) the presence of carbon; (3) a source of energy [1,2]. The presence of liquid water is favored because this molecule, very abundant in the Universe, has several interesting properties: its dipole moment makes it a very active solvent, allowing complex chemical reactions to take place (leading, in particular, to the formation of membranes); water is liquid over a large temperature range; moreover, its solid phase is lighter than the liquid, which makes possible the formation of an icy cover atop the ocean, protecting it in case of glaciation. We do not see any alternate molecule that could cumulate all these advantages. Carbon is favored because of its ability to form very strong $\mathrm{C}-\mathrm{C}$ bonds and to produce, thanks to its four valences, a great variety of chemical compounds, through carbon chains and ring structures. These properties explain why, in the interstellar medium, most complex molecules are based on carbon: the largest detected chain is $\mathrm{HC}_{11} \mathrm{~N}$, and many polycyclic aromatic hydrocarbons in the form $\mathrm{C}_{\mathrm{x}} \mathrm{H}_{\mathrm{y}}(\mathrm{PAHs})$ have also been found in the interstellar medium. Silicon could, in theory, be the basis of a similar chemistry; however, being heavier, it is ten times less abundant than carbon in the Universe; only a few silicon-based diatomic interstellar molecules have been detected so far. Finally, the solar radiation (or any stellar radiation) is the first natural possible energy source for life to develop; however, other sources can also be considered: the Urey-Miller experiment, leading to the synthesis of amino-acids, was first achieved in the presence of electrical discharges; geothermal energy (for instance in hydrothermal vents) could also be considered.

We will now concentrate on the first constraint, the presence of liquid water. Where can we find liquid water in planetary environments? The answer is given by the phase diagram of water (Figure $1)$.

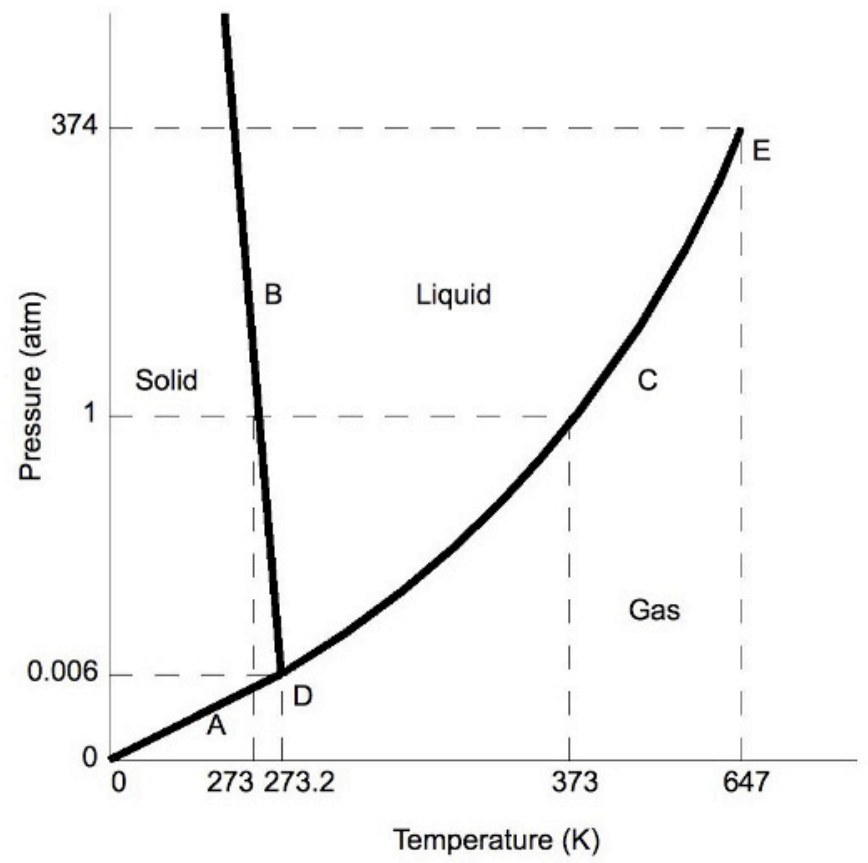

Fig. 1. The phase diagram of water ((C) ChemWiki (C) UC Davis, Wikimedia Commons). 
We can define, from this diagram, two kind of media where water can be liquid: (1) At moderate pressures and temperatures in the range $\left[0^{\circ}-100^{\circ} \mathrm{C}\right]$, we find the atmospheres of the rocky planets and possibly small exoplanets; (2) at higher pressures and temperatures, the interiors of outer icy satellites (and possibly exosatellites) can also host liquid water. In what follows, we will consider these different cases, from the solar system rocky planets to the outer satellites and finally the exoplanets.

\subsection{A possible diagnostic: The measurement of isotopic ratios}

Which diagnostics can we define for identifying living organisms in planetary environments? Chirality is a well-known property of living organisms, but the identification of chirality cannot be performed using remote sensing observations, nor with the in-situ instruments presently available on planetary landers or rovers. Another diagnostic can be used for (exo)planetary exploration: the measurement of isotopic ratios. In the case of carbon, the ${ }^{12} \mathrm{C} /{ }^{13} \mathrm{C}$ ratio can bring information about the nature of the organic material: On Earth, this ratio is 89 in the rocks, but higher (about 92) in living organisms. This difference is a result of photosynthesis, because the lighter isotope ${ }^{12} \mathrm{C}$ is used preferentially when $\mathrm{CO}_{2}$ is transformed under solar light into organics. The ${ }^{12} \mathrm{C} /{ }^{13} \mathrm{C}$ could be measured on other planets to search for this effect. Actually, the ${ }^{12} \mathrm{CH}_{4} /{ }^{13} \mathrm{CH}^{4}$ ratio could have been determined on Mars using the Tunable Laser Spectrometer experiment of the Sample Analysis (SAM) experiment aboard the Curiosity rover, if methane had been detected in sufficient abundances (over $20 \mathrm{ppb}$ ). Such an abundance had been tentatively reported from earlier observations (see [3] for a review), but no methane has been detected so far from Curiosity.

Another key measurement is the $\mathrm{D} / \mathrm{H}$ ratio in water. Indeed, this ratio increases at low temperatures, due to ion-molecule reactions, which enhance the abundance of the molecule bearing the heavier isotope (in the present case, $\mathrm{HDO}$ vs $\mathrm{H}_{2} \mathrm{O}$ ); such an effect is currently observed on many deuterated molecules, in the interstellar medium and also in the laboratory. In the solar system, the $\mathrm{D} / \mathrm{H}$ ratio is known to be enriched in ices, and the $\mathrm{HDO} / \mathrm{H}_{2} \mathrm{O}$ ratio is especially high in comets, formed in a very cold environment. A D/H ratio of $310^{-4}$, or twice the terrestrial value, has been measured on several comets coming from the Oort cloud. On Earth, the D/H measured in the terrestrial oceans (the "Standard Mean Ocean Water", or SMOW value) is $1.510^{-4}$, i.e. about 7 times higher than the protosolar value $\left(210^{-5}\right)$. This difference shows that the Earth atmosphere was not primarily outgassed from the globe, but mostly captured from meteoritic or cometary impactors, coming from colder regions of the outer solar system and enriched in deuterium. Did the terrestrial water come from asteroids or comets? Until recently, the outer main asteroidal belt was favored, because the $\mathrm{D} / \mathrm{H}$ measured in meteorites having parent bodies associated with this region was in agreement with the terrestrial value. However, a recent measurement, made by the Herschel satellite on a Kuiper Belt comet, the Jupiter-family comet $103 \mathrm{P} / \mathrm{Hartley} 2$, has led to a $\mathrm{D} / \mathrm{H}$ ratio in agreement with the SMOW value [4]. Could Kuiper Belt comets be responsible for the Earth's oceans? We should know know more about this question in 2014, when the Rosetta spacecraft, launched by the European Space Agency in 2004, encounters another Kuiper Belt comet, 67 P/Churyumov-Gerasimenko.

Another very different process may lead to strong isotopic fractionation of $\mathrm{D} / \mathrm{H}$ in water. In the atmospheres of terrestrial planets, $\mathrm{HDO} / \mathrm{H}_{2} \mathrm{O}$ is enriched by a factor over a hundred on Venus [5], and also, to a lesser extent, by a factor 5 to 6 on Mars [6]. The reason is a differential escape rate of water over the history of the two planets: $\mathrm{HDO}$, heavier than $\mathrm{H}_{2} \mathrm{O}$, escapes at a slightly slower rate. The high values of $\mathrm{D} / \mathrm{H}$ on Venus and Mars indicate that water was much more abundant in the primordial atmospheres of these planets, especially in the case of Venus. 


\section{The search for life on Mars}

Since its beginning, some forty years ago, the search for life on Mars has been the main driver for its space exploration. The Viking mission, launched in 1975, was explicitely designed for this purpose and, following the negative results concerning the detection of life on Mars, subsequent Martian missions were adjourned for about two decades. Over the past fifteen years, significant progress has been achieved about our understanding on the history of water on Mars, and the presence of liquid water at its surface, thanks to the findings of several orbiter and rover missions. Among the most exciting results are: with the MAG/ER experiment of Mars Global Surveyor, the discovery of a fossil remanent magnetic field in the oldest southern highlands, showing evidence for a dynamo effect and probably a magnetosphere in the first billion years of Mars' history [7]; the discovery of water under the polar caps from the Gamma Ray Spectrometer experiment aboard the Mars Odyssey mission [8]; the cartography of clays in ancient highlands and the detection of sulfates in chaotic regions by OMEGA/Mars Express, showing evidence for the presence of large amounts of liquid water in the early history of the planet, followed by more recent and localized episodic floods [9]. More recently, radar measurements by MARSIS aboard Mars Express and SHARAD aboard Mars Reconnaissance Orbiter have allowed us to better constrain the amount of water ice under the polar caps. Another new result of MARSIS is the map of the dielectric constant, which shows a lowemissivity region in the norther hemisphere, indicating the presence of porous sediments or a large water concentration [10]. The limits of this region are in remarkable coincidence with the shoreline shown by the laser altimeter of Mars Global Surveyor, which brings some support to the hypothesis of a past northern ocean (Figure 2).

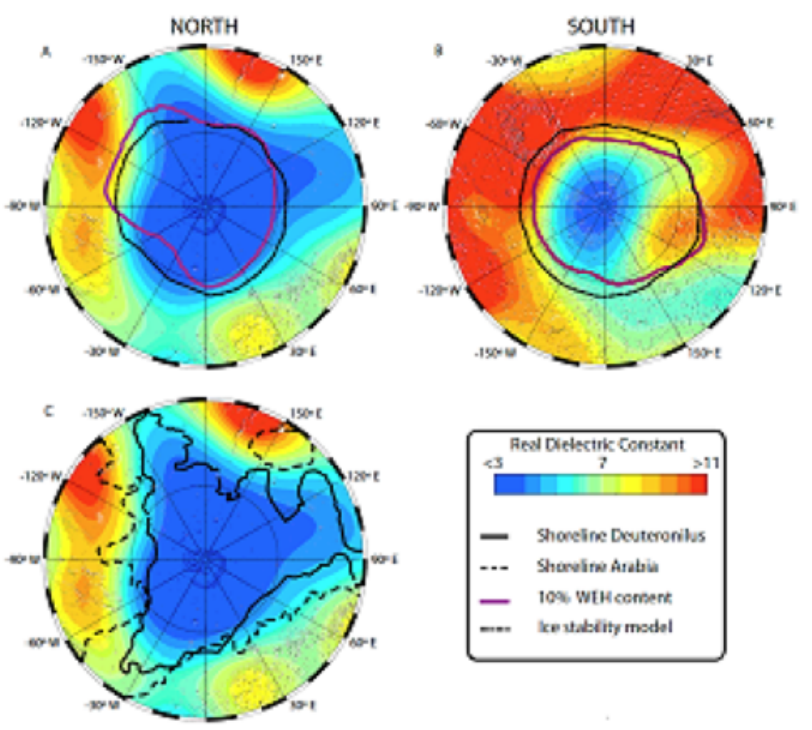

Fig. 2. The map of dielectric constant recorded by the MARSIS/Mars Express instrument. Left: Northern hemisphere; Right: Southern hemisphere. The shorelines are indicated on the lower left map. The figure is taken from Mouginot et al. 2012 [10].

Since August 2012, the Mars Science Laboratory rover of NASA, renamed Curiosity, is in operation in Gale crater, at the surface of Mars, with the objective of characterizing the geology, the mineralogy and the elemental abundances of the soil. Samples are collected at the surface or just below it, and analyzed in the SAM laboratory, with the main objective of searching for organic 
material and testing the past and present habitability of the planet. In the future, the space exploration of Mars will move on along several milestones: the MAVEN mission, to be launched by NASA in 2014, will determine the atmospheric escape of the planet; the ExoMars mission, led by Europe and Russia, will combine the Trace Gas Orbiter (TGO), to be launched in 2016, for characterizing the Martian atmospheric composition, and the ExoMars rover, to be launched in 2018, for an analysis of the Martian soil at deeper depths under the surface. In 2020, NASA plans to launch MSL-2020, a successor of Curiosity, as a preliminary step in view of a future Mars Sample Return mission.

\section{Outer satellites}

Our interest for outer satellites was strongly enhanced at the time of the Voyager flybys of the Galilean satellites in 1979, then Titan, in 1980 and 1981. Scientists discovered the active volcanism of Io, the possible water ocean under the surface of Europa, and the prebiotic molecules of Titan's atmosphere. From the viewpoint of exobiology, Europa and Titan were of major interest. The existence of a subsurface ocean in Europa opened the possibility of an environment where life might possibly appear; Titan was revealed as a possible laboratory for prebiotic chemistry, with, in addition, some analogy with planet Earth in terms of atmospheric composition and pressure (but, of course, not in terms of temperature).

These discoveries led to the design of the two subsequent main space missions toward the outer planets, Galileo and Cassini-Huygens. Galileo, launched by NASA in 1989, encountered Jupiter in 1995, sent a probe into its atmosphere and remained in operation around the Jovian system until 2003, making multiple flybys of the four Galilean satellites. Cassini-Huygens, jointly designed and operated by NASA and ESA, was launched in 1997, encountered Saturn in 2004, sent the Huygens descent probe down to Titan's surface in 2005; the Cassini orbiter is still in operation around Saturn's system today. In particular, Cassini has made an exciting discovery: the existence of an active cryovolcanism on Saturn's satellite Enceladus. The composition of Enceladus' plumes includes mostly water vapor but also nitrogen, methane and carbon dioxide [11]. The material outgassed from Enceladus appears to be the source of Saturn's E ring. Cryovolcanism was a priori unexpected on such a small satellite (Enceladus' diameter is about $500 \mathrm{~km}$ ) and its origin is still a matter of debate.

The case of Europa is of special interest because, unlike Ganymede and Callisto, which probably also host an internal liquid ocean, its liquid ocean is likely to reach the silicate bottom, making possible a complex chemistry at that interface. A recent reanalysis of the Galileo data has led to the conclusion that lakes might exist directly under the surface, at depths of a few kilometers only [12]. This result is based on the analysis of several icy domes, surprisingly tall (about 200 meters in altitude), in particular in the region of Conamara Chaos. These circular areas might have been formed from the breakup and refreezing of ice overlying extensive pockets of water beneath the surface (Figure 3). This result has important implications in terms of exobiology. Indeed, the depth of the icy crust is very uncertain, but could reach several tens of kilometers. Drilling through it would be practically unfeasible. Drilling down to a subsurface lake would still be a challenge, but possible conceivable for a future space exploration of the satellite. 


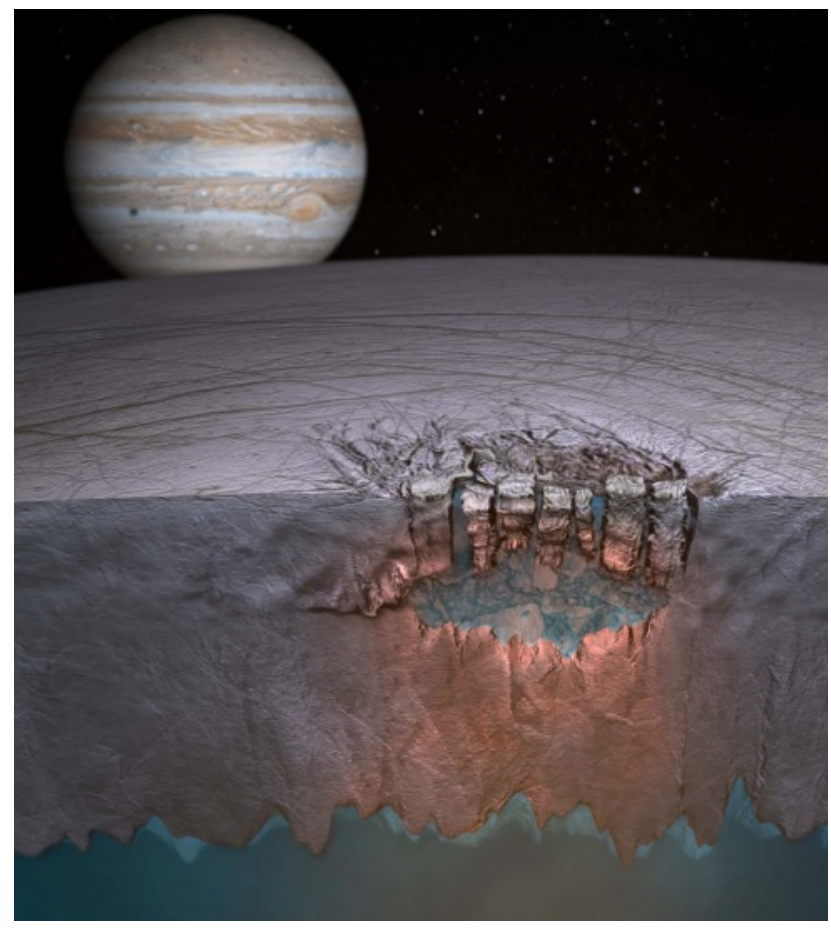

Fig. 3. This artist's picture shows a subsurface lake on Europa, as proposed by B. Schmidt et al. 2011 ([12]) (ㄷ Britney Schmidt / Dead Pixel VFX / Univ. of Texas at Austin).

The exploration of the Jovian system, as a possible witness of giant exoplanets' systems, and the indepth study of Galilean satellites, as possible targets for habitability, are the main scientific drivers of the future mission JUICE (Jupiter Icy Moons Explorer), recently approved by ESA for a launch around 2022, and an exploration of the Jupiter system in 2030 and beyond. First in orbit around Jupiter, the spacecraft, after several flybys of Callisto and Europa, will finally orbit Ganymede, for an in-depth exploration of the satellite's exosphere, surface and magnetic field. In the meantime, the JUNO mission, launched by NASA in 2011, will encounter Jupiter in 2016 and orbit the planet for an analysis of its internal composition and its magnetosphere, with the main objective of better understanding the giant planet's origin.

\section{How to search for habitable exoplanets?}

The discovery of extrasolar planets has been a revolution for astronomy. For millenia, philosophers and scientists had discussed their existence; since 1992, the first exoplanets have been identified, first around a pulsar [13], and later around a solar-type star [14]. This was already a major achievement; but the main surprise came from the nature of the giant exoplanets, discovered in the close vicinity of their host star. On the basis of the formation scenario of the solar system, where giant planets are formed far from the Sun, this situation was completely unexpected. Astronomers discovered that the solar system was not unique, but also that other planetary systems were very different from ours. 


\subsection{Exoplanet's characterization by transit spectroscopy}

Apart from the timing method, used to identify a planet around a pulsar from the analysis of its periodic radio signal, the first exoplanets have been detected by velocimetry: the method consists in measuring the relative motion of the host star, as seen from Earth, with respect to the center of gravity of the star-planet system. Since the beginning of the 2000s, a new method has been very powerful: the detection of exoplanets by transit. The presence of the planet is inferred from the small decrease of the stellar signal when occulted by the planet (about $1 \%$ for a Jupiter-size planet, and $10^{-4}$ for an Earthlike planet [15]). Ground-based surveys have been very fruitful for detecting giant exoplanets. For smaller exoplanets, more interesting from an exobiology viewpoint, space exploration was necessary. The CoRoT satellite, launched by CNES in 2006, and even more the Kepler satellite, launched by NASA in 2009, have opened a new era in the exploration of exoplanets. We now know almost 300 transiting exoplanets, and Kepler has identified over 2000 possible candidates [16]. The host stars of most of them are too weak for a confirmation by velocimetry, but their identification as candidates is important for statistical studies on the various populations of exoplanets.

The development of the transit method has opened a new field of research, now in full development: transit spectroscopy. Measurements were first achieved during primary transits (when the planet passes in front of the star). In this case, the atmosphere is observed at terminator (both morning and evening). Observations in the UV and visible range were recorded with the Hubble Space Telescope (detection of $\mathrm{H}$ I and $\mathrm{C}$ II in the UV, Na I, $\mathrm{H}_{2}$ Rayleigh scattering, haze in the visible). Molecular species were detected in the neutral atmosphere in the near infrared range $\left(\mathrm{H}_{2} \mathrm{O}, \mathrm{CH}_{4}, \mathrm{CO}_{2} ;\right.$ [17], Figure 4). Secondary transits (when the planet passes behind the star), provides us with a direct detection of the exoplanet's emission, and is also very useful for atmospheric characterization. In this case, the emission spectrum of the planet is observed on the dayside. Space data have been obtained with HSTNICMOS and with IRS and MIPS aboard Spitzer. Many articles have been published, leading to the (sometimes controversial) identifications of $\mathrm{H}_{2} \mathrm{O}, \mathrm{CH}_{4}, \mathrm{CO}$ and $\mathrm{CO}_{2}$. Most likely, spectroscopic observations using both primary and secondary transits are going to develop in the coming decade, as both techniques have their specific advantages and limitations.

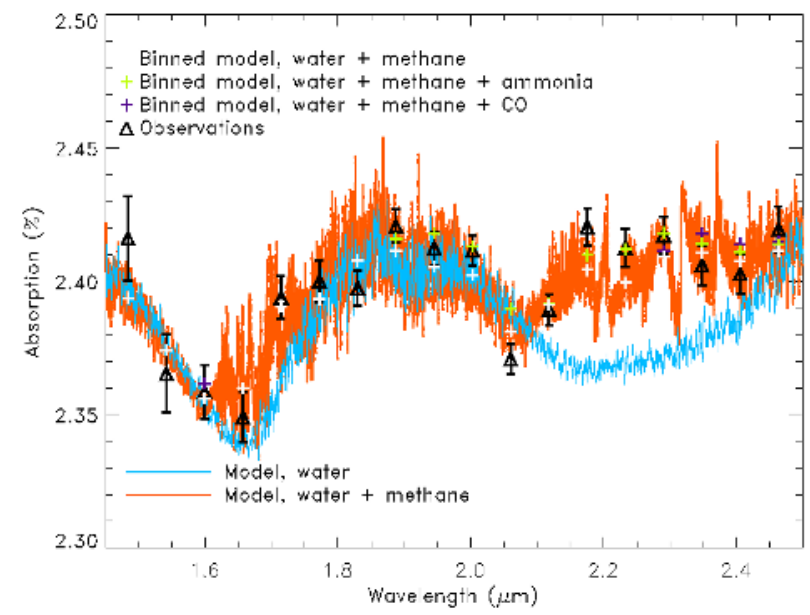

Fig 4. An example of primary transit spectroscopy on exoplanet HD189733b. The data (black points) are compared with two models including $\mathrm{H}_{2} \mathrm{O}$, with and without $\mathrm{CH}_{4}$ ). Comparison favors the presence of methane. The figure is taken from Swain et al. 2008 [17]. 


\subsection{How to search for habitable exoplanets?}

So far, for feasibility reasons, transit spectroscopy observations have been limited to hot Jupiters. For future observations, astronomers define the means, both from the ground and space, which will allow them to explore potentially habitable planets. What are the conditions to be fulfilled by these planets? A first category of objects must be able to host liquid water on their surface, i.e. to have an equilibrium temperature in the range of about $\left[0^{\circ} \mathrm{C}-100^{\circ} \mathrm{C}\right]$. The equilibrium temperature can be calculated from the fraction of stellar energy absorbed by the planet and converted into thermal heat (it depends upon the planet's albedo, for which an assumption is needed). For a given star, the region where water can be liquid defines a range of distances to the star called the habitable zone (HZ; figure 5). In the case of the Sun, it ranges from about 0.7 to 1.5 AU [18]. For brighter stars, the region extends at larger distances; for M-dwarf stars (a more interesting case, as these stars contribute to about $90 \%$ of the stellar population), it comes as close as $0.05-0.1 \mathrm{AU}$; these targets are especially suited for transit observations.

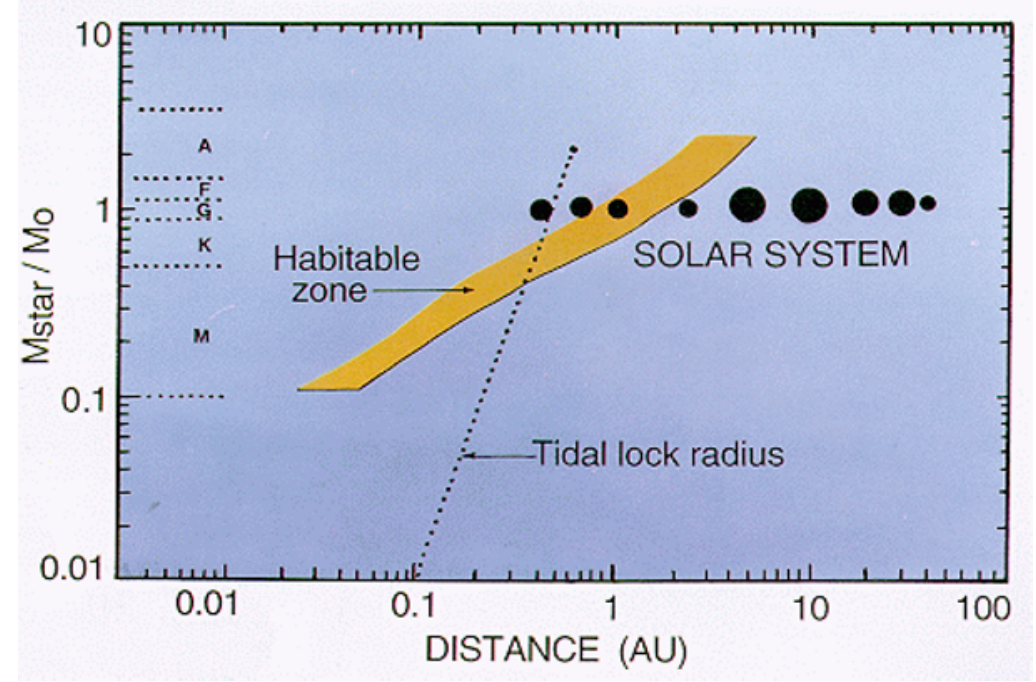

Fig. 5 The habitable zone for various types of stars. The dashed line shows the upper limit of the region where planets are expected to be tidally locked. (C) www.astro.psu.edu/users/williams).

What are the spectral signatures (the so-called «bio-markers ») which could be used to identify the presence of life on an exoplanet? Oxygen was first proposed because, in the absence of life, its presence on large amounts is not expected under thermochemical equilibrium [19]. Oxygen has a signature at $0.7 \mu \mathrm{m}$. Ozone was later suggested as a better tracer because its signature at $9.7 \mu \mathrm{m}$ is very strong, and can be observed with other key molecules $\left(\mathrm{CH}_{4}\right.$ at $7.7 \mu \mathrm{m}, \mathrm{CO}_{2}$ at $15 \mu \mathrm{m}, \mathrm{H}_{2} \mathrm{O}$ at $6.2 \mu \mathrm{m}, \mathrm{NH}_{3}$ at $10.5 \mu \mathrm{m}$ ), in a spectral range more favorable than the visible for spectroscopic characterization of exoplanets [20]. We should also mention the Red Vegetation Edge [21], a visible spectral signature marginally present on the Earth spectrum around $0.7 \mu \mathrm{m}$, although it will be probably difficult to detect on an exoplanet. 


\subsection{Future projects}

Several projects are presently in preparation or under development for exploring the atmospheres of potentially habitable exoplanets. Regarding ground-based observations, with the new generation of extremely large telescopes (ELTs) of 30 - 40 m diameter size, all equipped with the most performant adaptive optics systems, one can expect to gain a factor of about 10 to 16 in sensitivity with respect to the present largest telescopes, hence a factor 100 to 250 in exposure time, in the near infrared and mid infrared range. The number of exoplanets observable with transit spectroscopy will thus be drastically enhanced. There are presently two projects of telescopes of $30 \mathrm{~m}$ diameter or above: the Thirty Meter Telescope (TMT), in partnership between US and Canada, to be built at Mauna Kea Observatory, and the E-ELT (European Southern Observatory), with a diameter of 39 meters, to be built at Cerro Amazones in Chile. The first light of the E-ELT is planned for horizon 2022 and a suite of focal instruments is under development or under study [22].

In the case of space exploration, several projects are under study or under development. On the ESA side, the CHEOPS small mission, in partnership with Switzerland, has been approved by ESA in 2012. Its objective is to accurately measure the masses and radii of transiting exoplanets around bright stars, and to define suitable targets for future transit spectroscopy; the launch is scheduled for 2017. Still at ESA, two missions devoted to exoplanet exploration are submitted, among five candidates, as M3 medium missions: PLATO is a follow-up of CoRoT and Kepler with special focus on bright stars, also for selecting future targets for spectroscopy ; $E C h O$ is a mission designed for the transit spectroscopy of a large variety of exoplanets. The M3 selection is planned for the end 2013 and the launch is scheduled around 2022. On the NASA side, two projects devoted to exoplanets are in competition as Small Explorer missions: TESS is, like PLATO, a follow-up of Kepler oriented toward the detection of planets around nearby and brigh stars; FINESSE is, like EChO, a mission of spectroscopic characterization. The selection between these two projects, both precursors of the more ambitious PLATO and EChO missions, is expected in 2013 for a launch in 2016. Another important space project for exoplanets characterization is the James Webb Space Telescope (JWST). The successor of the HST, which includes a 6-m telescope and several infrared instruments, is planned for 2018. The NIRSpec instrument $(0.6-5 \mu \mathrm{m})$ and the MIRI instrument $(5-28 \mu \mathrm{m})$ will both be well suited for transit spectroscopy of exoplanets. Sensitivity calculations have been performed for various kinds of exoplanets [23].

In summary, with the ongoing space exploration program on Mars, the future JUICE mission toward outer icy satellites, and the various projects devoted to exoplanet characterization, one can hope significant progress in the decades to come regarding the exploration of planetary habitable environments. A new science, exoplanetology, is expected to emerge and develop, on interdisciplinary bases, gathering astronomers, planetologists, Earth science physicists, chemists and biologists. This mutidisciplinary approach has already started, especially in the frame of the EPOV programme of CNRS, and is expected to develop further in the coming decades.

\section{References}

1. I. Gilmour and M. A. Sephton, An Introduction to Astrobiology, Cambridge University Press (2004)

2. M. Ollivier, T. Encrenaz, F. Roques, F. Selsis, and F. Casoli, Planetary systems: Detection, Formation and Habitability of Extrasolar Planets, Springer (2009)

3. G. Villanueva, M. J. Mumma, R. E. Novak et al., Icarus, in press (2013)

4. P. Hartogh, D. Lis, D. Bockelée-Morvan et al., Nature 478, 218 (2011)

5. B. Bézard, C. de Bergh, D. Crisp and J.-P. Maillard. Nature 345, 508 (1990)

6. T. Owen, J.-P. Maillard, C. de Bergh and B. Lutz, Science 240, 1767 (1988)

01001-p.9 
7. M. H. Acuna, J. E. P. Connerney, N. F. Ness, R. P. Lin et al., Science 284, 790 (1999)

8. P. E. Boynton, W. C. Feldman, S. W. Squyres et al. Science 297, 81 (2002)

9. J.-P. Bibring, Y. Langevin, J. Mustard, F. Poulet et al. Science 312, 400 (2006)

10. J. Mouginot, A. Pommerol, P.,Beck et al. Geophys. Res. Letters 39, L02202 (2012)

11. D. Matson, J. C. Castillo, J. Lunine and T. V. Johnson, Icarus 187, 569 (2007)

12. B. Schmidt, D. D. Blankenship, G. W. Patterson, and P. M. Schenk, Nature 479, 502 (2011)

13. A. Wolszczan and D. A. Frail, Nature 355, 145 (1992)

14. M. Mayor and D. Queloz, Nature 378, 355 (1995)

15. M. Perryman, The Exoplanet Handbook, Cambridge University Press (2011)

16. N. M. Batalha, J. F. Rowe, S. T. Bryson et al. Astrophys. J. Supplement 204, id. 24 (2013)

17. M. Swain, G. Vasisht, and G. Tinetti, Nature 452, 329 (2008)

18. J. F. Kasting, D. P. Whitmire, and R. T. Reynolds, Icarus 101, 108 (1993)

19].T. Owen, In Strategies for the Search of Life in the Universe, Springer, Astrophysics and Space Science Library 83, 177 (1980)

20. J. R. P. Angel, A. Y. S. Cheng, and N. J. Woolf. Nature 322, 341 (1986)

21. S. Seager, E. L. Turner, J. Schafer et al. Astrobiology 5, 372 (2005)

22. M. Kissler-Patig and M. Luybenova, An expanding view of the Universe, European Southern Observatory Publication (2011, first ed. 2009)

23. S. Seager, D. Deming, and J. A. Valenti, in Astrophysics of the Next Decade: JWST and concurrent facilities, Astrophysics and Space Science Library, Springer Netherlands, p. 123 (2009) 